\title{
THE
}

\section{Weak biofilm formation among carbapenem-resistant Klebsiella pneumoniae}

Jaclyn A. Cusumano

University of Rhode Island

R. Caffrey

University of Rhode Island, aisling_caffrey@uri.edu

Kathryn E. Daffinee

Megan K. Luther

University of Rhode Island

Vrishali Lopes

Follow this and additional works at: https://digitalcommons.uri.edu/php_facpubs

The University of Rhode Island Faculty have made this article openly available. Please let us know how Open Access to this research benefits you.

This is a pre-publication author manuscript of the final, published article.

Terms of Use

This article is made available under the terms and conditions applicable towards Open Access Policy Articles, as set forth in our Terms of Use.

\section{Citation/Publisher Attribution}

Cusumano, J. A., Caffrey, A. R., Daffinee, K. E., Luther, M. K., Lopes, V., \& LaPlante, K. L. (2019). Weak biofilm formation among carbapenem-resistant Klebsiella pneumoniae. Diagnostic Microbiology and Infectious Disease, 95(4), 114877. doi: 10.1016/j.diagmicrobio.2019.114877

Available at: https://doi.org/10.1016/j.diagmicrobio.2019.114877 


\section{Authors}

Jaclyn A. Cusumano, R. Caffrey, Kathryn E. Daffinee, Megan K. Luther, Vrishali Lopes, and Kerry L. LaPlante 
1 Title: Weak biofilm formation among carbapenem-resistant Klebsiella pneumoniae

2 Running title: Klebsiella pneumonia biofilm formation

3 Abstract word count: 112

4 Manuscript word count: 1763

6 Jaclyn A. Cusumano ${ }^{1,2}$, Aisling R. Caffrey ${ }^{1,2,3}$, Kathryn E. Daffinee, ${ }^{1}$ Megan K. Luther ${ }^{1,2,3}$, Vrishali

7 Lopes ${ }^{1}$, Kerry L. LaPlante ${ }^{1,2,3,4}$

8

9 1. Infectious Diseases Research Program, Providence Veterans Affairs Medical Center,

10 Providence, RI, United States

11 2. College of Pharmacy, University of Rhode Island, Kingston, RI, United States

12 3. Center of Innovation in Long-Term Support Services, Providence Veterans Affairs Medical

13 Center, Providence, RI, United States

14 4. Warren Alpert Medical School of Brown University, Division of Infectious Diseases,

15 Providence, RI

17 Corresponding author: Kerry L. LaPlante, Pharm.D., FCCP, FIDSA, Professor, University of Rhode

18 Island, College of Pharmacy, 7 Greenhouse Rd, Suite 295A, Kingston, RI 02881, 401-874-5560 (office);

19 KerryLaPlante@uri.edu

22 Declarations of Interest: Kerry L. LaPlante receives research funding or is advisor/consultant

23 for Merck, Pfizer Pharmaceuticals, Ocean Spray Cranberries, Inc., Nabriva Therapeutics US, Inc.,

24 Melinta Therapeutics, Inc., and Tetraphase Pharmaceuticals. Aisling Caffrey has received

25 research funding from Pfizer, and Merck. Jaclyn A. Cusumano, Kathryn E. Daffinee, Vrishali

26 Lopes, and Megan K. Luther report no financial relationships. 


\section{Abstract}

28 Biofilm formation of multidrug and extensively drug resistant Klebsiella pneumoniae isolates is

29 poorly understood. We investigated 139 diverse clinical K. pneumoniae isolates that possess

30 various resistance patterns to evaluate the relationship between biofilm formation and resistance.

31 Antimicrobial resistance was compared among a diverse collection of weak versus strong biofilm-

32 forming K. pneumoniae, and predictors of strong biofilm formation were identified. Multi-drug

33 resistant isolates were more common among weak $(97.9 \%)$ versus strong biofilm formers $(76 \%$;

$34 \mathrm{p}=0.002$ ). Carbapenem-resistant $K$. pneumoniae were $91 \%$ less likely to form strong biofilm (odds

35 ratio $0.09 ; 95 \%$ confidence interval $0.02-0.33$ ). The statistically significant inverse relationship

36 between biofilm formation and antibiotic resistance suggests that virulence may be a trade-off for

37 survival.

39 Keywords: Klebsiella pneumoniae; biofilm; carbapenem resistance

40

41

42

43

44

45

46

47

48

49

50 


\section{INTRODUCTION}

53 Klebsiella pneumoniae, the most common and most concerning carbapenem-resistant

54 Enterobacteriaceae (CRE) (1), is associated with mortality rates up to $50 \%$ (2). Adding to this

55 challenging infection is $K$. pneumoniae's high propensity to form biofilms $(3,4)$. Biofilm-forming

56 K. pneumoniae are associated with foreign indwelling device related infections (4), as well as

57 urinary stones (5-7). The most common $K$. pneumoniae infections include urinary tract infections,

58 pneumonia, as well as intra-abdominal infections, which are prone to biofilm formation $(4,8)$.

59 Biofilm eradication requires high antimicrobial concentrations (9), which often cannot be

60 physiologically achieved in the blood stream or at the site of infection, thus potentially leading to

61 infection recurrence (4). Unfortunately, clinical microbiology labs cannot routinely test for biofilm

62 formation; however, tested phenotypic characteristics may help clinicians predict biofilm potential.

64 Multidrug-resistant (MDR) organisms have been associated with biofilm formation when

65 Klebsiella pneumoniae (10, 11), Staphylococcus aureus (11), Acinetobacter spp. (10, 11),

66 Pseudomonas aeruginosa (10,11), Escherichia coli $(10,11)$, coagulase-negative staphylococci

67 (10), or Enterococcus spp. (10) are assessed together $(10,11)$. However, the relationship

68 between biofilm-forming $K$. pneumoniae alone and antimicrobial resistance has not been fully

69 elucidated (12-14). The study objective was to determine whether certain antimicrobial class

70 resistance in K. pneumoniae was predictive of strong biofilm formation.

72 MATERIALS AND METHODS:

73 Our study included 139 unique K. pneumoniae clinical isolates obtained from the Centers for

74 Disease Control and Prevention (CDC; $n=66)$, Biodefense and Emerging Infections (BEl; $n=36$ ),

75 American Type Culture Collection (ATCC; $n=3$ ), and Providence Veterans Affairs (VA) Medical

76 Center and Rhode Island Hospital $(n=34)$. These isolates were selected because they are known

77 to be resistant to a range of antibiotic classes. Isolates from the Providence VA Medical Center 
were collected per the VA approved Institutional Review Board (IRB), Research and Development

79 (R\&D), and safety committee protocols.

80 A previously described biofilm assay was performed to assess biofilm formation (15-19). This

81 assay is considered the standard for evaluation of bacterial attachment and biofilm formation in

82 vitro (20). Isolates were obtained from culture stocks, stored at $-80^{\circ} \mathrm{C}$. After streaking on tryptic

83 soy agar and incubating for 18 to 24 hours, an inoculum of $6 \log _{10} \mathrm{CFU} / \mathrm{mL}$ in tryptic soy broth

84 with added $25 \mathrm{mg} / \mathrm{L}$ calcium, $12.5 \mathrm{mg} / \mathrm{L}$ magnesium, and $1.25 \%$ total dextrose (21). Media was

85 selected and validated based on the greatest biofilm formation, which is consistent with previously

86 published data for Escherichia coli (22) and Klebsiella pneumoniae $(17,18)$. Each isolate was

87 incubated in a 96-well plate (Costar 3596) for 24 hours at $37^{\circ} \mathrm{C}$ and $120 \mathrm{rpm}$ in octuplicate (17,

88 18). Wells were stained with $0.1 \%$ crystal violet (CV) and resolubilized with $33 \%$ glacial acetic

89 acid (19). K. pneumoniae (ATCC 700603) served as the biofilm positive control and media alone

90 was the negative control $(12,23-25)$.

91 Biofilm formation was measured as an optical density $\left(O D_{570}\right)$. We further categorized isolates

92 as either weak, moderate, or strong biofilm formers based on tertiles of $O D_{570 .}$ In order to assess

93 differences between the highest and lowest $O D_{570}$, we removed isolates in the moderate range

$94(19,26)$.

95 Organism susceptibility was obtained from the site of collection (eg. CDC, BEI). When results

96 were unavailable testing was performed by E-test or Kirby-Bauer disc diffusion on Mueller-Hinton

97 agar, and were interpreted according to 2017 CLSI susceptibility breakpoints $(27,28)$. The FDA

98 package insert for tigecycline (E-test MIC $\leq 2)(29)$ and EUCAST breakpoints for colistin (E-test

$99 \mathrm{MIC} \leq 2$ ) and fosfomycin (E-test MIC $\leq 32$ and disc diffusion $\geq 24$; both contained glucose-6-

100 phosphate) (30) were used as CLSI breakpoints were not available. Isolates were categorized

101 as multi-drug resistant (MDR), extensively drug-resistant (XDR), or resistant to specific 
102 antimicrobial classes/agents, according to CDC and European CDC expert consensus definitions

103 for Enterobacteriaceae (31). MDR isolates demonstrated non-susceptibility to at least one agent

104 in three or more antimicrobial categories out of 16 antimicrobial categories and XDR isolates

105 demonstrated susceptibility to at least one agent in less than or equal to two out of 16 antimicrobial

106 categories (31).

107 To assess the relationship between biofilm formation and resistance to specific antimicrobial

108 classes/agents, we grouped the 16 antimicrobial categories into 12 categories based on

109 mechanism of action (Table 1). Piperacillin/tazobactam and penicillin/ $\beta$-lactamase inhibitors were

110 grouped as penicillins plus $\beta$-lactamase inhibitors, and non-extended spectrum cephalosporins,

111 extended-spectrum cephalosporins, cephamycins, and ceftaroline were grouped as

112 cephalosporins. This allowed us to avoid collinearity in our statistical models due to overlap in

113 resistance between antimicrobial categories.

114 Differences in antimicrobial resistance among the weak and strong biofilm formation groups were 115 assessed with chi-square, Fisher's exact, or t-test as appropriate. Predictors of strong biofilm 116 formation were identified from a logistic regression model. A p-value of 0.1 was used for initial

117 inclusion in the model (Table 1) and stepwise backward elimination was used to identify 118 statistically significant predictors of strong biofilm formation (all $p$-values $<0.05$ ). We assessed 119 multicollinearity between potential predictors in the initial model from variance inflation factors,

120 and confirmed the absence of collinearity. A sensitivity analysis was conducted to identify 121 predictors of biofilm formation as a continuous measure $\left(\mathrm{OD}_{570}\right)$ using linear regression.

\section{RESULTS}

124 Optical density $\left(\mathrm{OD}_{570}\right)$ for all 139 isolates were divided using tertiles as follows: weak $(n=47$; $\left.125 \mathrm{OD}_{570} \leq 0.16\right)$, moderate $\left(n=46 ; 0.16<O D_{570}<0.59\right)$, and strong biofilm formers $\left(n=46 ; O D_{570} \geq\right.$ 126 0.59) (26). This method was internally validated as the positive control was consistently 
127 categorized as a strong biofilm former $\left(O D_{570} \geq 0.59\right)(26)$. Moderate isolates were removed for 128 a total cohort of 93 isolates (Table 1) to best predict biofilm formation extremes (26).

130 MDR isolates $(n=81)$ were more common among weak biofilm formers $(n=46,97.9 \%)$ versus

131 strong biofilm formers $(n=35,76.1 \% ; p=0.002)$, and XDR $(n=25)$ isolates were similar between

132 the groups ( $n=12,25.5 \%$ vs. $n=13,28.3 \% p=0.77)$. Number of resistant antimicrobial categories

133 and $\mathrm{OD}_{570}$ are shown in Figure 1. Resistance to all classes of beta-lactams (i.e. penicillins plus

$134 \quad \beta$-lactamase inhibitors, cephalosporins, monobactams, and carbapenems), aminoglycosides,

135 chloramphenicol, and fluoroquinolones were more common among weak biofilm formers

$136(\mathrm{p}<0.05)$. In the multivariate model, the only predictor of biofilm formation was carbapenem

137 resistance, which was inversely associated with strong biofilm formation (odds ratio, OR 0.09;

$13895 \%$ confidence interval, Cl 0.02-0.33). Therefore, carbapenem-resistant $K$. pneumoniae were

$13991 \%$ less likely to form strong biofilm.

141 As the proportion of XDR isolates did not vary between weak and strong biofilm formers, we 142 conducted a post-hoc sensitivity subgroup analysis excluding XDR isolates ( $\mathrm{n}=68)$ (Table 2).

143 Predictors of strong biofilm formation were again identified from a stepwise backward elimination 144 logistic regression model, with a p-value of 0.1 used for initial inclusion in the model (Table 2). 145 The only predictor of strong biofilm formation was the number of resistant categories, with an 146 odds ratio of $0.70(95 \% \mathrm{Cl} 0.56-0.86)$, where the odds of strong biofilm formation decreased by $14730 \%$ with each increase in the number of resistant categories.

149 In the sensitivity analysis of the continuous measure of biofilm formation, only fluoroquinolone 150 resistance was predictive of the $\mathrm{OD}_{570}$, with a parameter estimate of -0.44 and an intercept of 0.86 151 ( $p<0.001)$. According to this model, fluoroquinolone susceptibility had an $\mathrm{OD}_{570}$ of 0.86 , while 152 fluoroquinolone resistance had an $\mathrm{OD}_{570}$ of 0.42 . In other words, fluoroquinolone susceptible 
153 isolates were predicted to be strong biofilm formers, and fluoroquinolone resistant isolates were 154 predicted to be moderate biofilm formers.

\section{DISCUSSION}

157 This is the first study to our knowledge, to identify a statistically significant inverse relationship 158 between $K$. pneumoniae antimicrobial resistance and biofilm formation, where carbapenem-

159 resistant K. pneumoniae isolates were $91 \%$ less likely to be strong biofilm formers. Several 160 published studies have described higher biofilm formation in resistant $K$. pneumoniae isolates, 161 however, these descriptive studies did not assess whether resistance was predictive of biofilm 162 formation in multivariate analyses (10-14). This is also the first study to assess resistance and 163 biofilm formation of a diverse collection of $K$. pneumoniae isolates from multiple sources and 164 centers. Inclusion of isolates only from a single-center introduces potential bias if patients are 165 infected with the same organism, especially if isolates are from an outbreak (14). Additional 166 rationale for the difference in findings requires further research.

168 Potential limitations of our study include the overall resistance patterns of our isolates. The 169 majority of our isolates $(n=81,87.1 \%)$ were MDR, with the most isolates $(n=64,68.8 \%)$ resistant 170 to at least 12 out of 16 antimicrobial classes, but only 26.9\% $(n=25)$ were XDR. Inclusion of more

171 susceptible or XDR isolates may have resulted in different predictors of biofilm formation. As a 172 post-hoc sensitivity analysis we excluded XDR isolates, which support the findings from the weak 173 versus strong analysis, where strong biofilm formation was 30\% less likely with each increase in 174 the number of resistant categories.

175

176 Findings from previous studies may also be limited by misclassification of biofilm formation, which 177 may affect conclusions that biofilm formation is more common among resistant isolates. The 178 definition of biofilm formation varies across studies but the most common definition was originally 
179 described for Staphylococcus spp. $(15,16)$. This method utilizes an OD cut-off (ODc), defined as

180 three standard deviations above the average OD of the negative control, to determine biofilm

181 formation. Isolates are either non-adherent (OD $\leq \mathrm{ODc})$, weakly adherent (ODc $<\mathrm{OD} \leq 2 \times \mathrm{ODc})$,

182 moderately adherent $(2 \times O D c<O D \leq 4 x O D c)$, or strongly adherent $(4 x$ ODc $<O D)$.

183 Categorization by this method however, is limited when the negative control has a negligible OD

184 reading, which was the case for our study and previously described literature $(12,13)$. Applying

185 this method to our cohort, zero isolates were non-adherent, two weak, 12 moderate, and 125

186 strong biofilm formers. Therefore, we divided biofilm formation into tertiles (26), to overcome

187 potential bias of overestimating strong biofilm formation (12). Previous utilization of tertile biofilm

188 categorization was also utilized for $S$. aureus, however categorization should not be affected by 189 organism as our biofilm quantification method utilized was adapted for K. pneumoniae. We also

190 assessed biofilm formation as a continuous variable since there is no standard categorization.

191 However, interpretation of resulting odds ratios is challenging when compared to a dichotomous

192 variable of resistance versus susceptible, and is less clinically meaningful.

194 Optical density remains an indirect measurement of biofilm formation and standardized methods

195 for both quantification and categorization of K. pneumoniae biofilm formation are needed. Varied

196 definitions of biofilm formation are utilized, making direct comparisons across studies difficult. It

197 is imperative to standardize biofilm quantification to allow for accurate assessment of predictors

198 of biofilm across settings and to quantify the relationship between biofilm forming isolates and

199 clinical outcomes.

200

201 In our study, strong biofilm formation was $91 \%$ less likely with carbapenem-resistant $K$. 202 pneumoniae, which allows clinicians to better predict $K$. pneumoniae's ability to produce biofilm 203 by phenotypic resistance. This inverse relationship between biofilm formation and antibiotic 204 resistance suggests that virulence may be a trade-off for bacterial survival. 
205 Acknowledgements: The information provided in this manuscript are those of the authors and

206 do not necessarily reflect the position or policy of the United States Department of Veterans

207 Affairs. This work was supported in part, by the Office of Academic Affiliations (OAA) at the

208 Department of Veterans Affairs, and by the COIN: Center of Innovation in Long-Term Services

209 and Supports for Vulnerable Veterans; Providence, RI.

210

211 Funding: This work was unfunded and the authors received no outside funding for this work.

212

213

214

215

216

217

218

219

220

221

222

223

224

225

226

227

228 
231 1. Won SY, Munoz-Price LS, Lolans K, Hota B, Weinstein RA, Hayden MK, Centers for

232 Disease C, Prevention Epicenter P. 2011. Emergence and rapid regional spread of

233 Klebsiella pneumoniae carbapenemase-producing Enterobacteriaceae. Clin Infect Dis

$234 \quad 53: 532-40$.

235 2. Patel G, Huprikar S, Factor SH, Jenkins SG, Calfee DP. 2008. Outcomes of carbapenem236 resistant Klebsiella pneumoniae infection and the impact of antimicrobial and adjunctive 237 therapies. Infect Control Hosp Epidemiol 29:1099-106.

238 3. Ribeiro SM, Cardoso MH, Candido Ede S, Franco OL. 2016. Understanding, preventing 239 and eradicating Klebsiella pneumoniae biofilms. Future Microbiol 11:527-38.

240 4. Clegg S, Murphy CN. 2016. Epidemiology and Virulence of Klebsiella pneumoniae. $241 \quad$ Microbiol Spectr 4.

242 5. Schwaderer AL, Wolfe AJ. 2017. The association between bacteria and urinary stones. $243 \quad$ Ann Transl Med 5:32.

244 6. Nickel JC, Reid G, Bruce AW, Costerton JW. 1986. Ultrastructural microbiology of $245 \quad$ infected urinary stone. Urology 28:512-5.

$2467 . \quad$ Lebeaux D, Chauhan A, Rendueles O, Beloin C. 2013. From in vitro to in vivo Models of 247 Bacterial Biofilm-Related Infections. Pathogens 2:288-356.

248 8. De Rosa FG, Corcione S, Cavallo R, Di Perri G, Bassetti M. 2015. Critical issues for 249 Klebsiella pneumoniae KPC-carbapenemase producing K. pneumoniae infections: a 250 critical agenda. Future Microbiol 10:283-94. 
2519 9. Hoiby N, Bjarnsholt T, Moser C, Bassi GL, Coenye T, Donelli G, Hall-Stoodley L, Hola

252 V, Imbert C, Kirketerp-Moller K, Lebeaux D, Oliver A, Ullmann AJ, Williams C,

253 Biofilms ESGf, Consulting External Expert Werner Z. 2015. ESCMID guideline for the

254 diagnosis and treatment of biofilm infections 2014. Clin Microbiol Infect 21 Suppl 1:S1-

$255 \quad 25$.

256 10. Subramanian P, Shanmugam N, Sivaraman U, Kumar S, Selvaraj S. 2012. Antiobiotic

257 resistance pattern of biofilm-forming uropathogens isolated from catheterised patients in

258 Pondicherry, India. Australas Med J 5:344-8.

259 11. Sanchez CJ, Jr., Mende K, Beckius ML, Akers KS, Romano DR, Wenke JC, Murray CK.

2602013 . Biofilm formation by clinical isolates and the implications in chronic infections.

$261 \quad$ BMC Infect Dis 13:47.

262 12. Vuotto C, Longo F, Pascolini C, Donelli G, Balice MP, Libori MF, Tiracchia V, Salvia

263 A, Varaldo PE. 2017. Biofilm formation and antibiotic resistance in Klebsiella

264 pneumoniae urinary strains. J Appl Microbiol 123:1003-1018.

265 13. Yang D, Zhang Z. 2008. Biofilm-forming Klebsiella pneumoniae strains have greater

266 likelihood of producing extended-spectrum beta-lactamases. J Hosp Infect 68:369-71.

267 14. Bocanegra-Ibarias P, Garza-Gonzalez E, Morfin-Otero R, Barrios H, Villarreal-Trevino

268 L, Rodriguez-Noriega E, Garza-Ramos U, Petersen-Morfin S, Silva-Sanchez J. 2017.

269 Molecular and microbiological report of a hospital outbreak of NDM-1-carrying

$270 \quad$ Enterobacteriaceae in Mexico. PLoS One 12:e0179651.

271 15. Christensen GD, Simpson WA, Younger JJ, Baddour LM, Barrett FF, Melton DM,

272 Beachey EH. 1985. Adherence of coagulase-negative staphylococci to plastic tissue 
culture plates: a quantitative model for the adherence of staphylococci to medical

274 devices. J Clin Microbiol 22:996-1006.

275 16. Stepanovic S, Vukovic D, Hola V, Di Bonaventura G, Djukic S, Cirkovic I, Ruzicka F. 276 2007. Quantification of biofilm in microtiter plates: overview of testing conditions and 277 practical recommendations for assessment of biofilm production by staphylococci.

$278 \quad$ APMIS 115:891-9.

279 17. de Campos PA, Royer S, Batistao DW, Araujo BF, Queiroz LL, de Brito CS, Gontijo280 Filho PP, Ribas RM. 2016. Multidrug Resistance Related to Biofilm Formation in 281 Acinetobacter baumannii and Klebsiella pneumoniae Clinical Strains from Different 282 Pulsotypes. Curr Microbiol 72:617-27.

283 18. Silva A, Sousa AM, Alves D, Lourenco A, Pereira MO. 2016. Heteroresistance to colistin 284 in Klebsiella pneumoniae is triggered by small colony variants sub-populations within 285 biofilms. Pathog Dis 74.

286 19. Luther MK, Parente DM, Caffrey AR, Daffinee KE, Lopes VV, Martin ET, LaPlante KL. 287 2018. Clinical and Genetic Risk Factors for Biofilm-Forming Staphylococcus aureus. 288 Antimicrob Agents Chemother 62.

289 20. O'Toole GA. 2011. Microtiter dish biofilm formation assay. J Vis Exp doi:10.3791/2437.

290 21. Luther MK, Bilida S, Mermel LA, LaPlante KL. 2015. Ethanol and Isopropyl Alcohol 291 Exposure Increases Biofilm Formation in Staphylococcus aureus and Staphylococcus 292 epidermidis. Infect Dis Ther 4:219-26.

293 22. Naves P, del Prado G, Huelves L, Gracia M, Ruiz V, Blanco J, Rodriguez-Cerrato V, 294 Ponte MC, Soriano F. 2008. Measurement of biofilm formation by clinical isolates of 295 Escherichia coli is method-dependent. J Appl Microbiol 105:585-90. 
296 23. Naparstek L, Carmeli Y, Navon-Venezia S, Banin E. 2014. Biofilm formation and 297 susceptibility to gentamicin and colistin of extremely drug-resistant KPC-producing 298 Klebsiella pneumoniae. J Antimicrob Chemother 69:1027-34.

299 24. Liu C, Zhou Y, Wang L, Han L, Lei J, Ishaq HM, Nair SP, Xu J. 2016. Photodynamic 300 inactivation of Klebsiella pneumoniae biofilms and planktonic cells by 5 -aminolevulinic $301 \quad$ acid and 5-aminolevulinic acid methyl ester. Lasers Med Sci 31:557-65.

302 25. Baldiris R TV, Vivas-Reyes R, Montes A, Arzuza O. 2016. Anti-biofilm activity of 303 ibuprofen and diclofenac against some biofilm producing Escherichia coli and Klebsiella 304 pneumoniae uropathogens. Afr J Microbiol Res 10:1675-1684.

305 26. Guembe M, Alonso B, Lucio J, Perez-Granda MJ, Cruces R, Sanchez-Carrillo C, 306 Fernandez-Cruz A, Bouza E. 2017. Biofilm production is not associated with poor 307 clinical outcome in 485 patients with Staphylococcus aureus bacteraemia. Clin Microbiol $308 \quad$ Infect doi:10.1016/j.cmi.2017.10.018.

309 27. Anonymous. Clinical and Laboratory Standards Institute. Performance standards for 310 antimicrobial susceptibility testing: 27th informational supplement, (M100-S27). Wayne, $311 \quad$ PA: CLSI; 2017.

312 28. Anonymous. Clinical and Laboratory Standards Institute. Methods for dilution 313 antimicrobial susceptibility tests for bacteria that grow aerobically; approved standard: 314 tenth edition, (M07-A10):CLSI; 2015.

315 29. Tygacil. (tigecycline) [package insert]. Wyeth Pharmaceuticals Inc; Philadelphia $316 \quad(\mathrm{PA}): 2005$. 
317 30. Anonymous. The European Committee on Antimicrobial Susceptibility Testing. Breakpoint tables for interpretation of MICs and zone diameters. Version 7.1, 2017.

$319 \quad$ http://www.eucast.org.

320 31. Magiorakos AP, Srinivasan A, Carey RB, Carmeli Y, Falagas ME, Giske CG, Harbarth

321 S, Hindler JF, Kahlmeter G, Olsson-Liljequist B, Paterson DL, Rice LB, Stelling J,

322 Struelens MJ, Vatopoulos A, Weber JT, Monnet DL. 2012. Multidrug-resistant,

323 extensively drug-resistant and pandrug-resistant bacteria: an international expert proposal

324 for interim standard definitions for acquired resistance. Clin Microbiol Infect 18:268-81.

325

326

327

328

329

330

331

332

333

334

335

336

337

338

339

340

341 
342 Table 1. Klebsiella pneumoniae: antimicrobial resistance and biofilm formation

\begin{tabular}{|c|c|c|c|c|}
\hline Variable & $\begin{array}{l}\text { Total } \\
\text { Cohort } \\
(n=93)\end{array}$ & $\begin{array}{c}\text { Weak } \\
\text { Biofilm } \\
\text { Formation } \\
(n=47)\end{array}$ & $\begin{array}{c}\text { Strong } \\
\text { Biofilm } \\
\text { Formation } \\
\quad(n=46)\end{array}$ & $\mathrm{p}$-value \\
\hline $\begin{array}{l}\text { Number of Resistant Categories ( } n=16) \text {, } \\
\text { Median, (IQR) }\end{array}$ & $\begin{array}{c}13(11- \\
14)\end{array}$ & $13(12-14)$ & $11.5(3-14)$ & 0.01 \\
\hline Multidrug-resistant (MDR), n (\%)* & $81(87.1)$ & $46(97.9)$ & $35(76.1)$ & 0.002 \\
\hline Extensively drug-resistant (XDR), $n(\%)^{* *}$ & $25(26.9)$ & $12(25.5)$ & $13(28.3)$ & 0.77 \\
\hline $\begin{array}{l}\text { Penicillins + } \beta \text {-lactamase inhibitors, } \mathbf{n} \\
(\%)^{\neq}\end{array}$ & $79(84.9)$ & $46(97.9)$ & $33(71.7)$ & 0.0004 \\
\hline Cephalosporins, n (\%) & $82(88.2)$ & $46(97.9)$ & $36(78.3)$ & 0.003 \\
\hline Monobactam, n (\%) & $73(78.5)$ & $45(95.7)$ & $28(60.9)$ & $<0.0001$ \\
\hline Carbapenems, $\mathbf{n}(\%)^{\ddagger}$ \# & $70(75.3)$ & 44 (93.6) & $26(56.5)$ & $<0.0001$ \\
\hline Aminoglycosides, $\mathbf{n}(\%)$ & $72(77.4)$ & $43(91.5)$ & $29(63.0)$ & 0.001 \\
\hline Chloramphenicol, n (\%) & $65(69.9)$ & $38(80.9)$ & $27(58.7)$ & 0.02 \\
\hline Fluoroquinolones, n (\%) & 73 (78.5) & $45(95.7)$ & $28(60.9)$ & $<0.0001$ \\
\hline Tigecycline, n (\%) & $13(14.0)$ & $6(12.8)$ & $7(15.2)$ & 0.73 \\
\hline Tetracyclines, n (\%) & $44(47.3)$ & $23(48.9)$ & $21(45.7)$ & 0.75 \\
\hline Folate pathway inhibitor, $\mathbf{n}(\%)$ & $66(71.0)$ & $37(78.7)$ & $29(63.0)$ & 0.09 \\
\hline Fosfomycin, n (\%) & $61(65.6)$ & $29(61.7)$ & $32(69.6)$ & 0.42 \\
\hline Colistin, n (\%) & $11(11.8)$ & $8(17.0)$ & $3(6.5)$ & 0.12 \\
\hline
\end{tabular}

343 Bolded p-values indicate potential predictors of strong biofilm formation included in the initial

344 logistic regression model 
345 *MDR isolates demonstrated non-susceptibility to at least one agent in three or more

346 antimicrobial categories out of 16 antimicrobial categories

$347{ }^{*}$ XDR isolates demonstrated susceptibility to at least one agent in less than or equal to two out

348 of 16 antimicrobial categories

$349{ }^{\ddagger}$ penicillin $+\beta$-lactamase inhibitors category includes piperacillin/tazobactam and penicillin/ $\beta$ -

350 lactamase inhibitors

$351 \quad{ }^{\ddagger \neq}$ cephalosporins category includes non-extended spectrum cephalosporins, extended-

352 spectrum cephalosporins, cephamycins, and ceftaroline

353

354

355

356

357

358

359

360

361

362

363

364

365

366

367 
368 Table 2. Sensitivity analysis of non-XDR Klebsiella pneumoniae: antimicrobial

369 resistance and biofilm formation

\begin{tabular}{|c|c|c|c|c|}
\hline Variable & $\begin{array}{l}\text { Total } \\
\text { Cohort } \\
(n=68)\end{array}$ & $\begin{array}{c}\text { Weak } \\
\text { Biofilm } \\
\text { Formation } \\
(n=35)\end{array}$ & $\begin{array}{c}\text { Strong } \\
\text { Biofilm } \\
\text { Formation } \\
(n=33)\end{array}$ & $\mathrm{p}$-value \\
\hline $\begin{array}{l}\text { Number of Resistant Categories ( } n=16) \text {, } \\
\text { Median, (IQR) }\end{array}$ & $12(8-13)$ & $13(12-13)$ & $9(1-12)$ & $<0.0001$ \\
\hline Multidrug-resistant (MDR), $\mathbf{n}(\%)^{*}$ & $56(82.4)$ & $34(97.1)$ & $22(66.7)$ & 0.001 \\
\hline Extensively drug-resistant (XDR), $n(\%)^{\star \star}$ & 0 & 0 & 0 & --- \\
\hline Penicillins + $\beta$-lactamase inhibitors, $\mathbf{n}(\%)$ & $54(79.4)$ & $34(97.1)$ & $20(60.6)$ & 0.0002 \\
\hline Cephalosporins, $\mathbf{n}(\%)$ & $57(83.8)$ & $34(97.1)$ & $23(69.7)$ & 0.002 \\
\hline Monobactam, n (\%) & $48(70.6)$ & $33(94.3)$ & $15(45.5)$ & $<0.0001$ \\
\hline Carbapenems, n (\%) & $45(66.2)$ & $32(91.4)$ & $13(39.4)$ & $<0.0001$ \\
\hline Aminoglycosides, n (\%) & $48(70.6)$ & $32(91.4)$ & $16(48.5)$ & 0.001 \\
\hline Chloramphenicol, n (\%) & $40(58.8)$ & $26(74.3)$ & $14(42.4)$ & 0.008 \\
\hline Fluoroquinolones, $\mathbf{n}(\%)$ & $48(70.6)$ & $33(94.3)$ & $15(45.5)$ & $<0.001$ \\
\hline Tigecycline, n (\%) & $6(8.8)$ & $4(11.4)$ & $2(6.1)$ & 0.67 \\
\hline Tetracyclines, n (\%) & $20(29.4)$ & $12(34.3)$ & $8(24.2)$ & 0.36 \\
\hline Folate pathway inhibitor, n (\%) & $41(60.3)$ & $25(71.4)$ & $16(48.5)$ & 0.053 \\
\hline Fosfomycin, n (\%) & $41(60.3)$ & $19(54.3)$ & $22(66.7)$ & 0.30 \\
\hline Colistin, n (\%) & $6(8.8)$ & $4(11.4)$ & $2(6.1)$ & 0.44 \\
\hline
\end{tabular}

370 Bolded $p$-values indicate potential predictors of strong biofilm formation included in the initial

371 logistic regression model 
372 *MDR isolates demonstrated non-susceptibility to at least one agent in three or more

373 antimicrobial categories out of 16 antimicrobial categories

$374{ }^{* *}$ XDR isolates demonstrated susceptibility to at least one agent in less than or equal to two out

375 of 16 antimicrobial categories

$376{ }^{\ddagger}$ penicillin $+\beta$-lactamase inhibitors category includes piperacillin/tazobactam and penicillin/ $\beta$ -

377 lactamase inhibitors

$378 \quad{ }^{\ddagger \neq}$ cephalosporins category includes non-extended spectrum cephalosporins, extended-

379 spectrum cephalosporins, cephamycins, and ceftaroline

380

381

382

383

384

385

386

387 
Figure 1. Klebsiella pneumoniae biofilm formation and resistance $(n=139)$

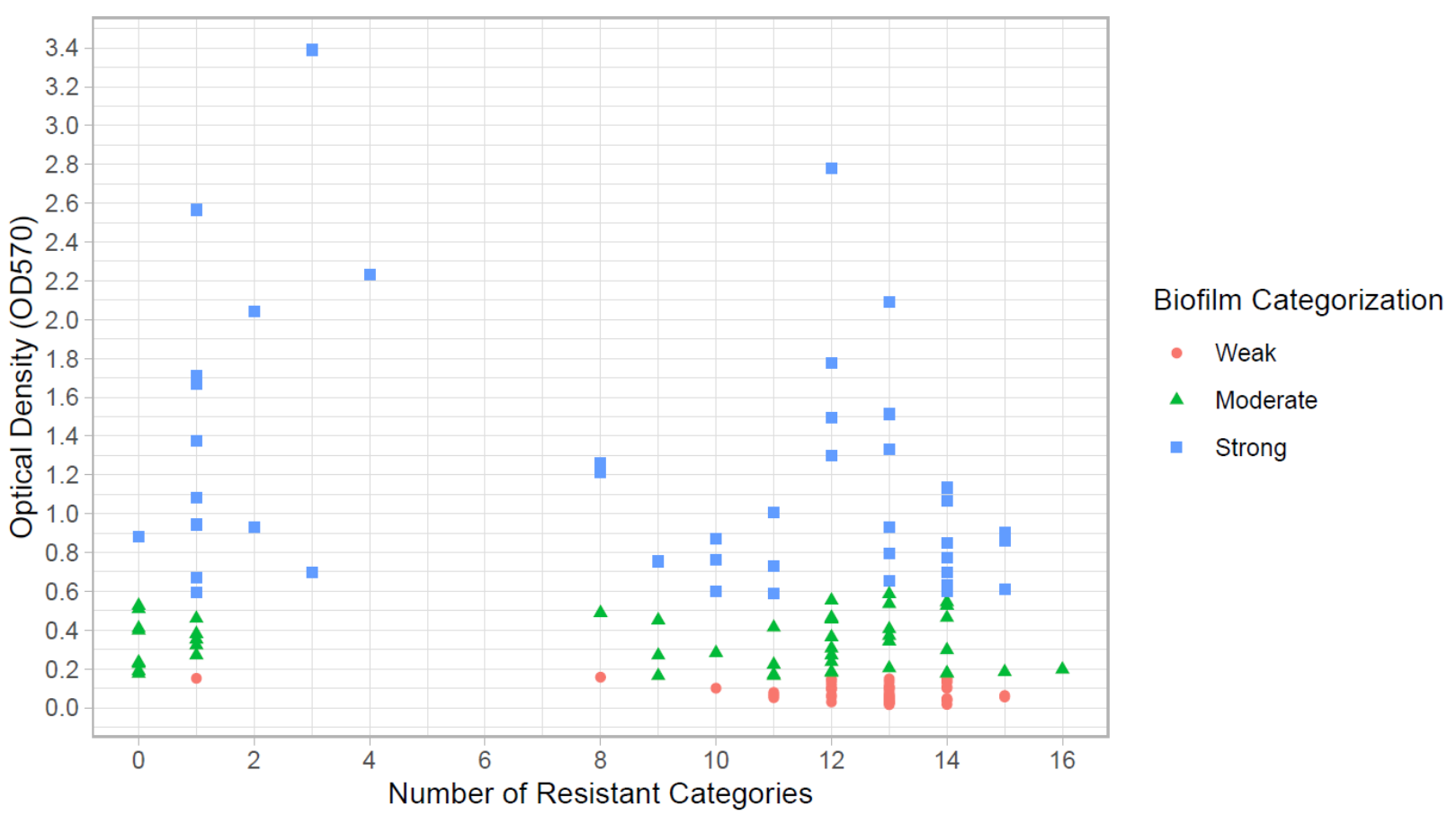

388

389 (2 column fitting image) 